International Journal of Engineering \& Technology, $7(2.21)(2018) 283-287$
International Journal of Engineering \& Technology
SPC
Website $:$ www.sciencepubco.com/index.php/IJET
Research paper

\title{
Traffic accident monitoring system using deep learning
}

\author{
A. Manikandan ${ }^{*}$, R. Anandan ${ }^{2}$ \\ ${ }^{l}$ Department of Computer Science \& Engineering, Vels Institute of Science, \\ Technology \& Advanced Studies(VISTAS), Chennai, India. \\ ${ }^{2}$ Department of Computer Science \& Engineering, Vels Institute of Science, \\ Technology \& Advanced Studies(VISTAS), Chennai, India. \\ *Corresponding author E-mail: mani.se@velsuniv.ac.in
}

\begin{abstract}
A short time period in development of rural places and public vehicle transportation system globally increased. The road accident are increased by the traffic problems last five years. It is a big problem of human society. These traffic accident are how can we happen and how to solve traffic management. Here we collect the traffic accident data and GPS record data using these data to build a deep learning model of stochastic gradient descent learning algorithm method used to solve critical problem of a traffic accident risk.
\end{abstract}

Index Terms: Deep learning, GPS, stochastic gradient descent.

\section{Introduction}

The Transport system in the modern cities are well developed and continue to growth. Transport vehicle are rapidly increased in modern cities. these growing Transportation system are creating a problem of Traffic jams and traffic accident are day by day increased. Nowadays we improve our transport system using GPS monitoring and surveillance cameras, these are used to predict the traffic monitoring. now everyone use the android mobile to create the application for traffic locating and the vehicle moving system. the peak time traffic data and moving vehicle information with GPS record to show able the drives to verify the traffic locating information and choose to less traffic locality information and choose to less traffic area to predict traffic jams. In India collecting the report of traffic accident data year wise released by Indian health organization 2016.The data said every year increasing the traffic accident and affecting most of them injured and died. What reasons to traffic accident means drivers activity, signal problem, proper road and weather condition. These reasons are finding and analyzing to reduce the traffic accident rate. Here we collect large amount of traffic data in India last five years 2012 to 2016 traffic accident data around 24lakhs in the traffic monitoring system to build a deep learning model structure use to predict traffic accident data.

\section{System overview}

In this method using deep learning of every single data to be analyzed in the system model. Here collecting the big and heterogeneous data of traffic accident data and GPS record data to be added in the system deeper model the user can be access the data in the way of input to the system model and pass the value to a instructions steps to move the data collection portion. In the data collection collect the expect data to the user. Next to the steps pass on the data analysis. This steps to analyzed the user needs data to be analyzed from deeper model. The deeper architecture provide the correct locating of traffic accident data and user change the route of traffic less areas. The user want to moving the traffic less areas and free from the driving tension.

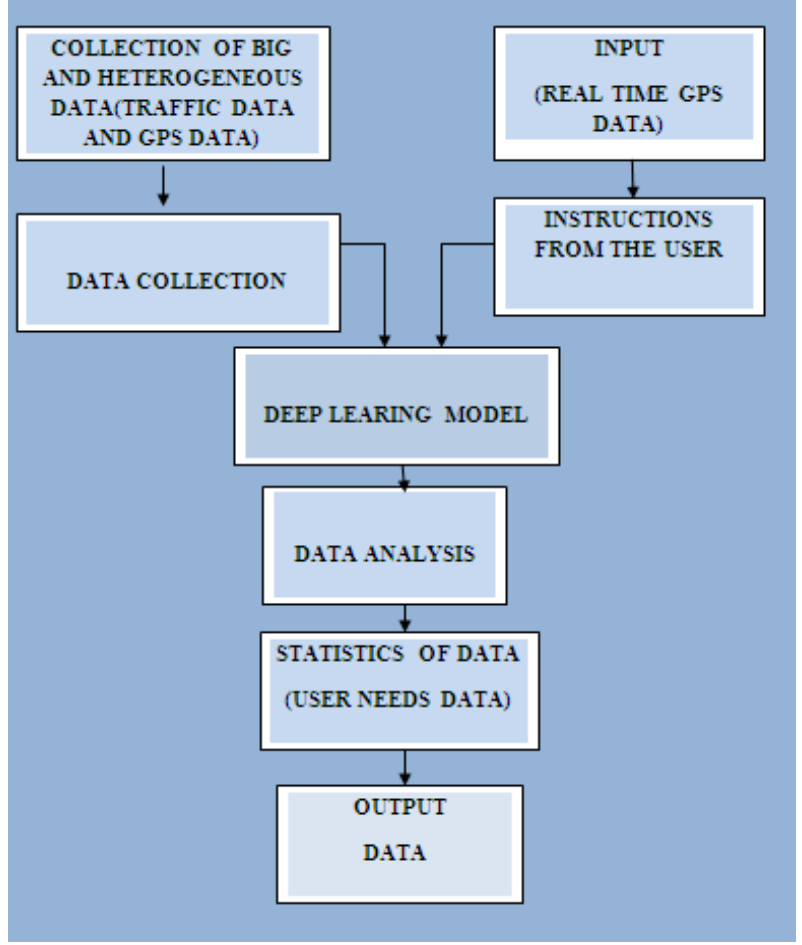

Figure 1: System overview model

The driver gets exact data and travel along the data. Customers reduce the stress of traffic jams. In that model to be executed the traffic jams are easily located and change of traffic less route areas. It is helpful to the drivers GPS monitoring and passing the easiest travelling way of traffic less routes to be used. 


\section{Deep learning overview}

Learning is the process knowing about the new one. In that learning different types of visual, logical ,solitary ,physical ,verbal and social learning. These are the basic learning method learn something new and different. Here using the deep learning find the neural networks. In that learning learn by several layer of nodes. both input and output can be analyzed layer by layer in that deep learning method. Here four types of deep learning unsupervised networks, pertained networks, convolutional neural networks, recurrent neural networks and recursive neural networks. just as our brains seem to. we have always had good algorithms for learning the weights in networks with 1 hidden layer but these algorithms are not good at learning the weights for networks with more hidden layers. reminder/quick-explanation of how neural network weights are learned the idea of unsupervised feature learning (why 'intermediate features' are important for difficult classification tasks, and how NNs seem to naturally learn them) The 'breakthrough' - the simple trick for training Deep neural networks. Deep learning, it is used to learn a complex data problems. it is access a hierarchically represents the abstract data. Complex and abstract data passing through the different stages of layer to be transformed. deep learning mainly focuses in that Learning from the order high level to low level data. For example recoginizing words from audio, and object form the images, and poses video form the movements this reason for the movement of deep learning. Advantages of data tagging, image and speech recogizition, information retrieval, natural languages processing. These algorithms are used most popular, there are deep boltzman machine, restricted boltzman machine, deep belief networks, convolutional, stacked auto encoder. Deep boltzman machine is used to layer by layer learning.it is used to finding the multiple hidden layers. Restricted boltzman machine, no visible unit and no hidden, In that no visible unit is not connected to any other visible unit structure. Here no hidden unit data is not connected to the other hidden unit. Restricted boltzman machine is used as that raw data into hidden units. reconstruction of errors. Deep belief networks is used to learn multilayer of randomly learning the data and hidden variables are detector. Convoutional neural networks is used to high level image to low level image can be classified. Convolutional layers are three types convolutional layer, pooling layer, dense layer. Convolutional layer is used specified convolutional filter used for splitting the data. Pooling layer is used to reduce the dimensionality of the pixels. Dense layer is used check the connection between every node to every node in the layer. Stacked auto encoder is used to capture the structure of input data to rebuild the input in the output layer. These are learning algorithm are described. Here used stochastic descent deep learning algorithm are used to predict the traffic accident risk.

\section{Implementation of deep learning}

Here any optimization needs, our main idea is to reduce the relating to the function .a particular way that relating task calculate the comparison of the true getting result and the assuming the related result. this method we will be using the objective function and loss function described.

$\min _{x} \sum_{i=1}^{N} f\left(x ; y_{i}\right)$

We must need to get the real fact of resulting data to be reduced. here using the method of deep learning used to predicting the exacted result of the data. stochastic gradient descent method is used repeating the process of analyzing the traffic monitoring and get the exacted result of the function. Previously using the target is less than areas to predict function. but now increasing the access areas and getting the true result with the help of this method.

Large scale optimization

$h(x)=\frac{1}{N} \sum_{i=1}^{N} f\left(x ; y_{i}\right)$ here entire data cannot be used to calculate of this method. Because layer by layer analysis the data. computing the gradient takes o(n) time.

$\nabla h(x)=\frac{1}{N} \sum_{i=1}^{N} \nabla f\left(x ; y_{i}\right)$

Gradient descent with big data

$\nabla h(x)=\frac{1}{2 N} \sum_{i=1}^{N} \nabla f\left(x ; y_{i}\right)+\frac{1}{2 N} \sum_{i=1}^{N} \nabla f\left(x ; y_{i}\right)$

every iteration can be used to select a data in a randomized. here data are spreading in a traffic layer accessing the data one by one analyzed.

Step 1.Estimate a starting design $\mathrm{x}(0)$ and set the iteration counter $\mathrm{k}=0$. Select a convergence parameter $\varepsilon>0$

Step 2. Calculate the gradient of $\mathrm{f}(\mathrm{x})$ at the point $\mathrm{x}(\mathrm{k})$ as . $c^{(k)}=\nabla f(x(k))$ calculate $\|\mathrm{c}\|=\sqrt{c^{T} c} .\|\mathrm{c}\|<\varepsilon$ then stop the iteration process as $\mathrm{x}^{*}=\mathrm{x}(\mathrm{k})$ is a minimum point. Otherwise, go to Step 3.

Step 3. Let the search direction at the current point $x(k)$ as $d(k)=-$ $\mathrm{c}(\mathrm{k})$.

Step 4. Calculate a step size $\alpha(\mathrm{k})$ to minimize $\mathrm{f}(\mathrm{x}(\mathrm{k})+\alpha(\mathrm{k}) \mathrm{d}(\mathrm{k})$. A one-dimensional search is used to determine $\alpha(\mathrm{k})$.

Step 5. Update the design as $x(k+1)=(x(k)+\alpha(k) d(k)$.. Set $k=k+1$ and go to Step 2.

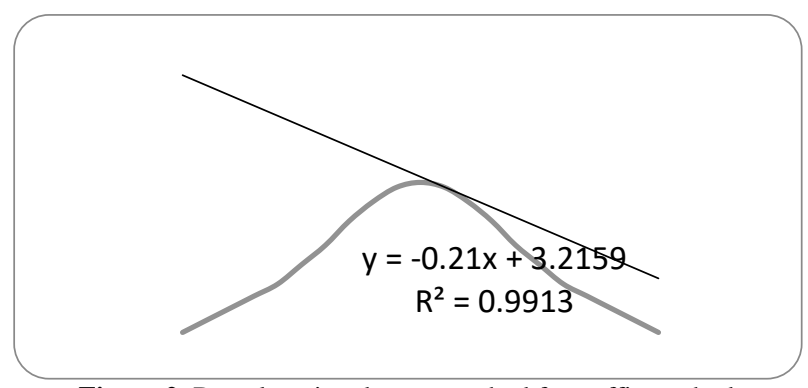

Figure 2: Deep learning descent method for traffic method.

The algorithm for descent understood this is only an approximation of the real gradient but it can be proven that we will order to reach the minimum by following this gradient.

There are many advantages to using Stochastic gradient Descent learning algorithm can be summarizing in follows,

$x_{t+1}=x_{t}-\alpha \nabla f\left(x_{t} ; y_{\tilde{i t}}\right)$

$E\left[x_{t+1}\right]=E\left[x_{t}\right]-\alpha E\left[\nabla \mathrm{f}\left(x_{t} ; y_{i t}\right)\right]$

$=E\left[x_{t}\right]-\alpha \frac{1}{N} \sum_{i=1}^{N} \nabla f\left(x_{t} ; y_{i}\right)$

Stochastic gradient descent convergence

$x_{t+1}-x^{*}=x_{t}-x^{*}-\alpha\left(\nabla h\left(x_{t}\right)-\nabla h\left(x^{*}\right)\right)-$

$\alpha\left(\nabla f\left(x_{t} ; y_{i t}\right)-\nabla \mathrm{h}\left(x_{t}\right)\right) \quad=\left(I-\alpha \nabla^{2} h\left(z_{t}\right)\right)\left(x_{t}-x^{*}\right)-$ $\alpha\left(\nabla f\left(x_{t} ; y_{i t}\right)-\nabla h\left(x_{t}\right)-(8)\right.$

Stochastic gradient descent convergence

$$
\begin{gathered}
\operatorname{Var}\left(x_{t+1}-x^{*} \mid x_{t}\right) \\
=\operatorname{Var}\left(\left(I-\alpha \nabla^{2} h\left(z_{t}\right)\right)\left(x_{t}-x^{*}\right)-\alpha\left(\nabla f\left(x_{t} ; y_{i t}\right)\right.\right. \\
\left.\left.-\nabla h\left(x_{t}\right)\right) \mid x_{t}\right) \\
=\operatorname{Var}\left(\alpha\left(\nabla f\left(x_{t} ; y_{i t}\right)-\nabla h\left(x_{t}\right)\right) \mid x_{t}\right) \\
=\alpha^{2} \operatorname{Var}\left(\nabla f\left(x_{t} ; y_{i t}\right)-\nabla h\left(x_{t}\right) \mid x_{t}\right) \\
=\alpha^{2} E\left[\left\|\nabla f\left(x_{t} ; y_{i t}\right)-\nabla h\left(x_{t}\right)\right\|^{2} \mid x_{t}\right]
\end{gathered}
$$

Stochastic gradient descent convergence

$E\left[\left\|x_{t+1}-x^{*}\right\|^{2} \mid x_{t}\right]=\left\|E\left[x_{t+1}-x^{*} \mid x_{t}\right]\right\|^{2}+\operatorname{Var}\left(x_{t+1}-\right.$ $\left.x^{*} \mid x_{t}\right) \leq(1-\alpha \mu)^{2}\left\|x_{t}-x^{*}\right\|^{2}+\alpha^{2} M($ for $\alpha \ll 1)$ 
$E\left[\left\|x_{t+1}-x^{*}\right\|^{2}\right] \leq(1-\alpha \mu)^{2} E\left[\left\|x_{t}-x^{*}\right\|^{2}\right]+\alpha^{2} M$

the stochastic gradient descent algorithm

Steps as follows,

Step1: Stochastic Gradient Descent

Initialize

$\omega_{1}$ for $\mathrm{k}=1$ to $\mathrm{K}$ do

Sample an observation i uniformly at random

Update

end for

$$
\omega_{k+1} \longleftarrow \omega_{k}-\alpha \nabla f_{i} \omega_{k}
$$

Return $\omega_{k}$

step 3 Parallel SGD

Shuffle the data . this is an expensive operation

for $\mathrm{k}=1$ to $\mathrm{K}$ do . in parallel

Perform SGD on the $\mathrm{k}$ th random partition of the data, producing model $\omega_{k}$

end for

Compute the average of the models $\omega=\frac{1}{k} \sum k \omega_{k}$

Return $\omega$

step 4 Lloyd's iteration (k-means algorithm)

Start with an arbitrary* set of k cluster centers, $\mathrm{c}_{1}{ }^{(1)}, \ldots, \mathrm{c}_{\mathrm{k}}{ }^{(1)}$

for $\mathrm{t}=1, . . \mathrm{T}$ do

1. Assignment step: Assign each point to closest center.

$$
\begin{gathered}
c_{i}^{(t)}=\left\{x:\left\|x c_{i}^{(t)} \quad\right\| 2 \leq\left\|x-c_{\ell}^{(t)}\right\| 2 \forall \ell=1, \ldots k\right\}, i \\
=1 \ldots k
\end{gathered}
$$

2. Update step: Update cluster centers by averaging set of points assigned to each cluster.

$$
c_{i}^{(t+1)}=\frac{1}{c_{i}^{(t)}} \sum_{x \in c^{t}} x, i=1 \ldots k
$$

end for

step 5 Distributed k-means

Start with an arbitrary* set of $\mathrm{k}$ cluster centers,

$\mathrm{c}_{1}{ }^{(1)}, \ldots, \mathrm{c}_{\mathrm{k}}{ }^{(1)}$ for $\mathrm{t}=1, \ldots, \mathrm{T}$ do

1) Broadcast current model: $\left\{c_{1} \ldots c_{k}\right\}$. small: $k$ vectors of length $d$

2) Assignment step: Do an RDD map to find closest center to each point; emit key-value

pairs (cluster ID, point).

3) Update step: reduce By Key with cluster ID as the key to compute average of data points

within each cluster.

end for

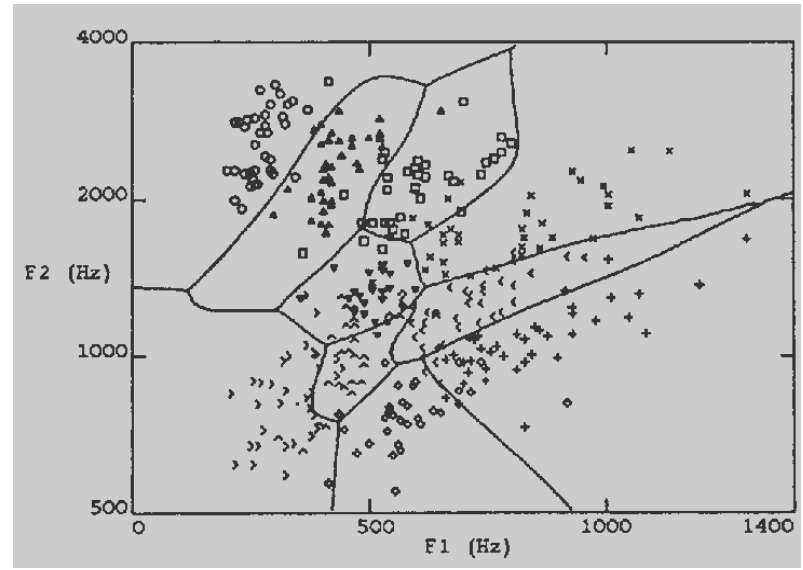

Figure 3: Traffic less and high areas using deep learning

In that figure stochastic gradient learning algorithm used to locate the traffic less and more areas are analyzed in that model. Drivers gets the information from this method to avoid traffic jam and struggled. Here using the identification of specified marks layer by layer analyzed and choose the best way to track and travel along with the location. that image enlarged way to find areas affecting traffic areas minimum and maximum of areas affecting the traffic location. Here $\mathrm{f} 1$ and $\mathrm{f} 2$ frequency method of 0 to $2000 \mathrm{~Hz}$ monitoring areas of traffic jams. Dark symbol shown the crowd of heavy traffic jams in that location. little bit darken bubble shown the less traffic jams. Total method can accessed the way of easy route to any struggle travel.

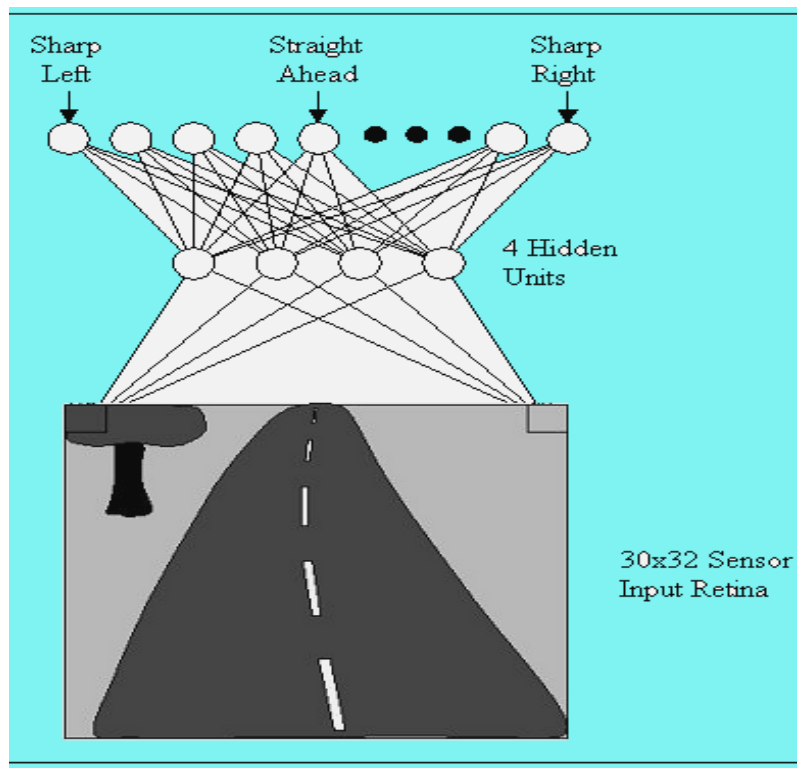

Figure 4: Vehicle sensor using deep learning

In that vehicle sensor access the road figure and find unwanted disturbance of travelling monitoring and track shortest way to reach the location. Here need get the total amount of data put in the main memory. the traffic data can be he. it can be handle difficult. between access the related data. Previously accessing data did not easily get. so update the data layer and layer. so here using the deep learning of stochastic method to find the data at a time one by one. Here we collected very large data and different data to understand. user mobility will struggle road accident risk. the pre proposal user mobility data for crashes occur

Between moving transport and people. we mining these data affected by other critical factors. stochastic gradient

Descent learning for predictable using collection of user and traffic monitoring data to find the shortest path in the road ways of without struggling in the vehicle traffic jams. here more number of data collected from the various location of accident data and monitoring data. these data to used to deep training model structure can be created.in this model can be transfer the information to grid data. grid data is used to transfer the distributed data to a training model.

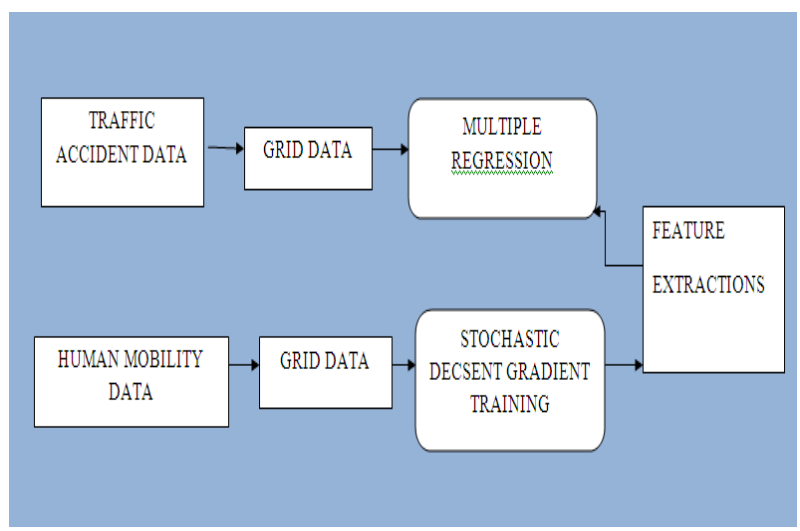

Figure 5: Stochastic descent traffic accident training model

The data grid can be analyzed by using regression model. it is a statistical method to analyzed among variable similarity .our real data is a multivariable. It is analysis a tool for all factors. it can be the number of vehicles detected between the two particular slices. that human access that to enlarge the traffic areas of the data. stochastic gradient method analyzed the grid data to be meaning full structure of the feature extraction. 


\section{Huge Amount of data}

Here we collect the data from Indian government website during the year 2013 to 2016 road accident data state wise. large data and evaluated as following in the year 2013 traffic accident occur in india overall state wise 486476 lakhs people can be affected.2014 overall state wise 489400 lakhs people suffered.2015 in india 501423 lakhs people can be affected. in the year 2016 state wise 480652 lakhs people can be suffered in the road accident.

\section{INDIA -STATE WISE ROAD ACCIDENT DATA 2013 TO 2016}

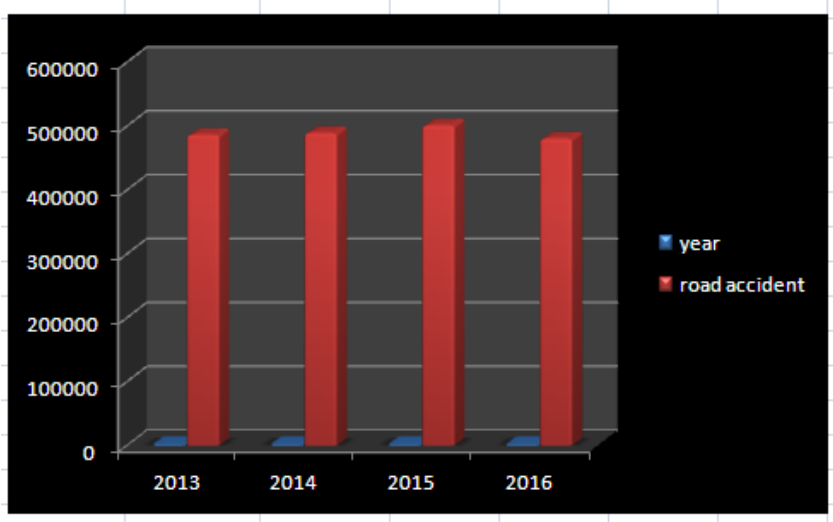

Figure 6: India state wise road accident data

\section{Result and discussion}

We choose the time quantum slices to access the traffic monitoring data to give the driver for handling traffic jams. Here select the half hour with 48 slices in the traffic areas in one day.

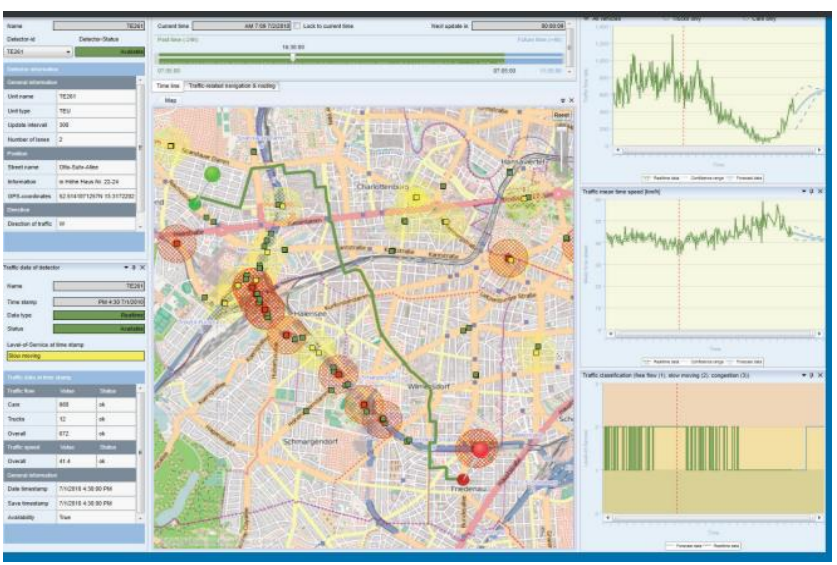

Figure 7: Deep traffic monitor various areas

one day fully checking the traffic areas using the deep learning method to find the shortest path in the way of travel. In that image shows the traffic areas using the colors red and green. Green color indicates the traffic less areas, red color shows traffic rush areas to be indentified.

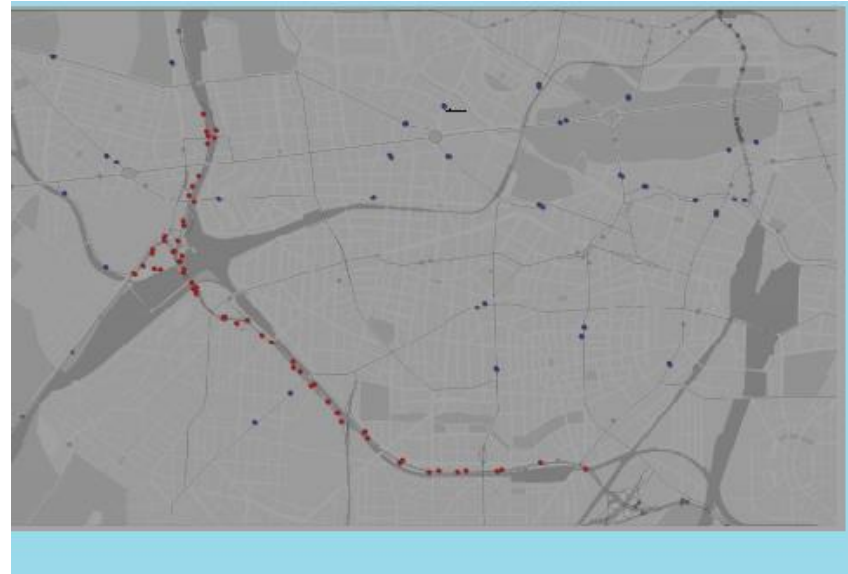

Figure 8: Deep sensing of traffic areas

In that image can show the city traffic sensing model. Here also same indentified method of traffic areas using red and green pixels. Red pixel indentified traffic rush areas displayed. Green pixel indicates traffic less areas. It is used less traffic areas find and travel along that road.E

\section{Related work}

Presently, a number of researches on analyzing traffic accident have been proposed, mainly focusing on hot spot finding the area of traffic accident location. Previously, Using stack denoise encoder method to used predict the stages of traffic location. In this denoise encoder used to locating areas but did not find the particular location of data. So, we move on that stage using method of stochastic gradient descent learning used to monitoring the areas layer by layer accessing the traffic accident data.

\section{Conclusion}

The concept of this paper, huge collected data and different data in road traffic accidents and driver data mobility in Chennai, it has been collected. By mining these huge collected data, how users data affects traffic accident. Here analyzed a deep learning model to extract the features to user monitoring data, and accessed a simple traffic predict model for analyzing traffic accident risk in huge collecting data in a real-time. It is used to aware people for less traffic areas to find travel along that road. These research data explore in our model efficiency. However, our study has several limitations to the traffic accidents. Users sensing data, these data are cannot to constructing a good method for the total risks prediction. In future exact traffic area data using to improve the present model.

\section{References}

[1] Eagle N, Pentland AS \& Lazer D, "Inferring friendship network structure by using mobile phone data", Proceedings of the National Academy of Sciences, Vol.106, No.36, (2009), pp.15274-15278.

[2] Fan Z, Song X \& Shibasaki R, "Cityspectrum: a non-negative tensor factorization approach", Proceedings of the ACM International Joint Conference on Pervasive and Ubiquitous Computing, (2014), pp.213223

[3] Grover A, Kapoor A \& Horvitz E, "A deep hybrid model for weather forecasting", Proceedings of the21th ACM SIGKDD International Conference on Knowledge Discovery and Data Mining, (2015), pp.379-386.

[4] Hinton GE \& Salakhutdinov RR, 'Reducing the dimensionality of data with neural networks", Science, Vol.313, No.5786, (2006), pp.504-507.

[5] Hinton G, Deng L, Yu D, Dahl GE, Mohamed AR, Jaitly N, Senior A, Vanhoucke V, Nguyen P, Sainath TN \& Kingsbury B, "Deep neural networks for acoustic modeling in speech recognition: The shared views of four research groups", Signal Processing Magazine, Vol.29, No.6, (2012), pp.82-97. 
[6] Hinton GE, Osindero S \& The YW, "A fast learning algorithm for deep belief nets", Neural computation, Vol.18, No.7, (2006), pp.1527-1554.

[7] Huang W, Song G, Hong H \& Xie K, "Deep architecture for traffic flow prediction: Deep belief networks with multitask learning", IEEE Transactions on Intelligent Transportation Systems, (2014). 